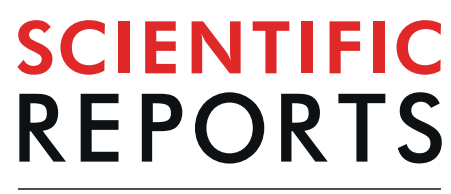

natureresearch

Check for updates

\title{
Fluorescence spectroscopy and chemometrics for simultaneous monitoring of cell concentration, chlorophyll and fatty acids in Nannochloropsis oceanica
}

\author{
Marta Sá ${ }^{1,2}$, Carlo G. Bertinetto ${ }^{3}$, Narcís Ferrer-Ledo ${ }^{2}$, Jeroen J. Jansen ${ }^{3}$, Rene Wijffels ${ }^{2}$, \\ João G. Crespo ${ }^{1}$, Maria Barbosa² \& Claudia F. Galinha ${ }^{1 凶}$
}

Online monitoring of algal biotechnological processes still requires development to support economic sustainability. In this work, fluorescence spectroscopy coupled with chemometric modelling is studied to monitor simultaneously several compounds of interest, such as chlorophyll and fatty acids, but also the biomass as a whole (cell concentration). Fluorescence excitation-emission matrices (EEM) were acquired in experiments where different environmental growing parameters were tested, namely light regime, temperature and nitrogen (replete or deplete medium). The prediction models developed have a high $\mathrm{R}^{2}$ for the validation data set for all five parameters monitored, specifically cell concentration (0.66), chlorophyll (0.78), and fatty acid as total $(0.78)$, saturated $(0.81)$ and unsaturated $(0.74)$. Regression coefficient maps of the models show the importance of the pigment region for all outputs studied, and the protein-like fluorescence region for the cell concentration. These results demonstrate for the first time the potential of fluorescence spectroscopy for in vivo and real-time monitoring of these key performance parameters during Nannochloropsis oceanica cultivation.

Microalgae industrial production is still a niche industry, although efforts are being done to improve the economic viability of the overall process ${ }^{1,2}$. Online monitoring through spectroscopic techniques is already a reality in several other bio-based industries ${ }^{3-6}$. Development of an appropriate tool able to monitor several metabolites simultaneously would be a great advantage. Nowadays, biological parameters are monitored off-line, where a sample is withdrawn, from the cultivation or from the process in the biorefinery, to be analysed. These analyses can take some minutes or a couple of hours, or days, depending on the parameter to be measured and techniques involved. For example, cell concentration is a rather fast and simple method, while assessing metabolites' content is much slower, because extraction steps and chromatographic techniques are often needed. Fluorescence spectroscopy represents a viable solution since it is a non-invasive and non-destructive technique that already proved its value in other industries, like the food industry ${ }^{3-5}$, or even in wastewater treatment plants that are characterised for being complex biological systems $\mathrm{s}^{6-8}$. This technique is able to detect natural fluorophores but is also sensitive to interactions between fluorophores and non-fluorescent compounds, increasing the range of compounds that can be monitored by it ${ }^{9}$. Additionally, with the improvement of chemometric tools, it is possible to extract more information from such signals.

Nannochloropsis oceanica is a promising microalga due to its ability to produce high amount of lipids. This microalga is cultivated in sea water, which has been pointed as the most sustainable solution for microalgae production $^{2,10,11}$. The most common product of a microalgae industry is still the whole biomass, being of extreme importance to monitor cell concentration ${ }^{12}$. It is also known that chlorophyll is an important pigment in several

${ }^{1}$ LAOV-REQUIMTE, Chemistry Department, FCT, Universidade Nova de Lisboa, Caparica, Portugal. ${ }^{2}$ Bioprocess Engineering, AlgaePARC, Wageningen University and Research, Wageningen, Netherlands. ${ }^{3}$ Radboud University, Department of Analytical Chemistry and Chemometrics, Institute for Molecules and Materials, Nijmegen, Netherlands. ${ }^{\circledR e}$-mail: cf.galinha@fct.unl.pt 


\begin{tabular}{|l|l|l|}
\hline Temp $\left({ }^{\circ} \mathbf{C}\right)$ & Nitrogen supply $^{(\text {a) }}$ & Light cycle (hours) \\
\hline 15 & X & d $(24)$ \\
\hline 20 & X & d (24) \\
\hline $25^{(\mathrm{b})}$ & X & d $(24)$ \\
\hline 25 & X & d $/$ n $(16 / 8)$ \\
\hline 25 & $\sqrt{ }$ & d/n $(16 / 8)$ \\
\hline $25 \rightarrow 15$ & $\sqrt{ }$ & d/n $(16 / 8)$ \\
\hline 30 & X & d $(24)$ \\
\hline
\end{tabular}

Table 1. Experimental conditions of the eight batch experiments performed. Three different environmental growing parameters were tested, namely temperature, nitrogen supply. $\left({ }^{(a)} \mathrm{X}=\right.$ absent; $\sqrt{ }=$ present;) and light cycle (d (24): $24 \mathrm{~h}$ of light; or d/n (16/8): $16 \mathrm{~h}$ of light and $8 \mathrm{~h}$ of dark). ${ }^{(\mathrm{b})}$ This batch was performed twice.

industries ${ }^{13,14}$, and being a oleaginous microalga, there are an increasing interest in industrializing $N$. oceanica to produce high quantities of lipids, but also high nutritional value oils ${ }^{2,10}$.

Previous studies demonstrated the advantage of using fluorescence spectroscopy in a microalgae biorefinery context using Dunaliella salina ${ }^{15-17}$. This microalga is also a saline microalga capable of producing high contents of carotenoids, being already produced at industrial scale for it. Fluorescence spectroscopy coupled with chemometric tools was used to develop prediction models for several parameters, such as cell concentration and viability, carotenoids content and nitrate concentration for $D$. salina upstream processes ${ }^{15-17}$. Cell concentration of Chlorella vulgaris and Spirulina sp. was also determined by Shin et al. using a in situ fluorometry technique ${ }^{18}$.

More recently, a new study from the authors, showed that eicosapentaenoic acid (EPA) can be also monitored using fluorescence spectroscopy in N. oceanica cultures ${ }^{19}$. Therefore, the goal of this work was to develop models to predict simultaneously cell concentration, chlorophyll and fatty acid content in N. oceanica, based only on fluorescence spectra acquired directly from in vivo culture broths. Furthermore, the spectral regions with higher relevance for the prediction models of all parameters were also studied, providing a better understanding of how the fluorescence can be useful to monitor fluorophores and non-fluorophores molecules. The development of this monitoring tool, able to be used online and based on a non-destructive technique will enhance the knowledge about the culture at real time, during the cultivation process, contributing for an increase competitiveness of microalgae industry.

\section{Material and Methods}

Nannochloropsis oceanica pre-culture and experiments. Nannochloropsis oceanica NCT02 was provided by NECTON, S.A. (Olhão, Portugal). The $N$. oceanica inoculum was kept in $250 \mathrm{~mL}$ Erlenmeyer flasks, under the follow conditions: $25^{\circ} \mathrm{C}, 90 \mathrm{rpm}$ in an orbital shaker, $100 \mu \mathrm{mol} / \mathrm{m}^{2}$.s of incident light, day/ night cycle (16/8 hours) light regime, and $0.2 \% \mathrm{CO}_{2}$. The cultivation media contained natural sea water (from Eastern Scheldt, the Netherlands) filtered $(0.2 \mu \mathrm{m})$ and supplemented with $10.7 \mathrm{mM}$ of $\mathrm{NaNO}_{3}, 0.535 \mathrm{mM}$ of $\mathrm{KH}_{2} \mathrm{PO}_{4}$. NUTRIBLOOM from PhytoBloom, a nutrient solution, and HEPES buffer $(20 \mathrm{mM})$ were added to the media and the $\mathrm{pH}$ set to 7.8. Medium sterilization was performed by cellulose acetate membrane filtration (using SARTOBRAN Capsule with $0.2 \mu \mathrm{m}$ of pore size, Sartorius) directly into the Erlenmeyer or bioreactor. Experiments were performed in batch mode, in a heat-sterilized flat-panel, with a $1.8 \mathrm{~L}$ of working volume and a light path of $20.7 \mathrm{~mm}$ (Labfors $5 \mathrm{Lux}$, Infors HT, Switzerland, 2010). The light was provided by LED lamps (28 V, 600 Watt) with warm spectrum $(450-620 \mathrm{~nm})$. In the beginning of the experiment light was set at $200 \mu \mathrm{mol} / \mathrm{m}^{2} . \mathrm{s}$, and increased to $636 \mu \mathrm{mol} / \mathrm{m}^{2}$.s when the back light reached $50 \mu \mathrm{mol} / \mathrm{m}^{2}$. The culture homogenization was done by filter sterilized air in an airlift-loop at a flow rate of $1 \mathrm{~L} / \mathrm{min}$, and the $\mathrm{pH}$ was controlled by $\mathrm{CO}_{2}$ injection. The bioreactor temperature was controlled by water-jacket.

Three cultivation parameters were tested in eight experiments (Table 1): light regime, temperature, and nitrogen supply - with $(\sqrt{ })$ or without $(\mathrm{X})$ nitrogen. Light regime was set in the beginning of the experiment and two approaches were tested: 24 hours of light or $16 \mathrm{~h}$ of light and $8 \mathrm{~h}$ of dark $(\mathrm{d} / \mathrm{n}$ cycle). Temperature was set in the beginning $\left(15,20,25\right.$ and $\left.30^{\circ} \mathrm{C}\right)$ and kept through the experiment; in one batch, the temperature was decreased from 25 to $15^{\circ} \mathrm{C}$ when a light supply rate of $1 \times 10^{-13} \mu \mathrm{mol} / \mathrm{cell} . \mathrm{s}$ was reached. All batches started with a replete nitrogen medium to enable biomass growth. For six of the eight batches (Table 1, Nitrogen supply "X"), a second step, the nitrogen depletion phase, was performed. Briefly, the biomass was collected, centrifuged $(2500 \mathrm{rpm}$, 15 minutes) and washed with nitrogen deplete medium, and the bioreactor was then refilled with culture and nitrogen deplete medium until reaching a specific light supply rate of $1 \times 10^{-13} \mu \mathrm{mol} / \mathrm{cell}$.s. More detailed information about the experiments are available in Sá et al. ${ }^{19}$.

Offline measurements. Samples were taken every day to measure cell concentration, chlorophyll content, fatty acid composition and spectrofluorescence.

Cell concentration was measured in a MULTISIZER II (Beckman Counter), in duplicates, using a $50 \mu \mathrm{m}$ aperture tube and Isotone II diluent to dilute the samples.

Chlorophyll content was assessed by a spectrophotometric method as described by Leu and $\mathrm{Hsu}^{20}$. An aliquot of $2 \mathrm{~mL}$ was centrifuged $(5000 \mathrm{~g}, 5 \mathrm{~min})$ and stored at $-80^{\circ} \mathrm{C}$ until further analysis. Extraction was performed with $2 \mathrm{~mL}$ of methanol, samples were sonicated for $5 \mathrm{~min}$ and incubated for $40 \mathrm{~min}$ at $60^{\circ} \mathrm{C}$, following by cooling for $15 \mathrm{~min}$ in ice. Extraction steps were repeated until a white pellet was recovered. The modified Arnaud equation was used to calculate chlorophyll content: 


$$
\text { Chla }=\left(16.72 \times A_{665}-9.16 \times A_{652}\right) \times \text { dilution factor }(m g / L)
$$

Lipid composition was measured in lyophilized biomass samples, previously washed with $0.5 \mathrm{M}$ ammonium formate, as described by Breuer et al. and Leon-Saiki ${ }^{21,22}$. Briefly, $10 \mathrm{mg}$ of sample were disrupted by beat beater and an extraction was performed with chloroform:methanol (1:1.25, v-v), containing the internal standards for triacylglycerol (TAG) and polar (PL) fractions, $170 \mu \mathrm{g} / \mathrm{mL}$ of tripentadecanoin (9:0) and $170 \mu \mathrm{g} / \mathrm{mL}$ of 1,2-dipentadecanoyl-sn-glycero-3-[phosphor-rac-(1-glycerol)] (sodium salt) (15:0) respectively. TAG and PL were then separated in a SPE silica gel column (Sep-Pak Vac 6cc, Waters) using hexane:diethylether (7:1, v-v) and methanol:acetone:hexane $(2: 2: 1, \mathrm{v}-\mathrm{v})$ respectively. Methylation was performed in both fractions prior to quantification by gas chromatography (GC-FID). The results were calculated as percentage of total, saturated and unsaturated fatty acids in a dry weight basis.

Fluorescence spectra were acquired in a Shimadzu RF-6000 spectrofluorophotometer. The samples were placed in a cuvette and no sedimentation was observed during the spectra acquisition, which occurred in 5 minutes. The excitation-emission matrices (EEMs) obtained ranged from 250 to $790 \mathrm{~nm}$ for excitation wavelengths, and between 260 and $800 \mathrm{~nm}$ for emission wavelengths, in steps of $5 \mathrm{~nm}$. Excitation and emission monochromator slit widths were set at $3 \mathrm{~nm}$, with a scan speed of $12000 \mathrm{~nm} / \mathrm{min}$.

Chemometric models development. The EEMs obtained during the eight experiments were combined and pre-processed together using drEEM toolbox (http://www.models.life.ku.dk/dreem) ${ }^{23}$. Rayleigh scatter of first order was removed and replaced by empty values; the second order was replaced with an interpolation of surrounding data point ${ }^{23}$. Any fluorescence signal corresponding to emission wavelengths ( $y$-axis) shorter than the excitation wavelengths ( $\mathrm{x}$-axis) was replaced by zeros. Inner filter effects, i.e. whenever excessive concentration causes re-absorption within the sample and thus a non-linear fluorescence response, were accounted for using a correction matrix derived from the sample's absorbance spectrum ${ }^{24}$.

The pre-processed EEMs were correlated with five biological parameters (cell concentration, chlorophyll and fatty acids content as total, saturated and unsaturated) using Projection to Latent Structures (PLS) regression. This method finds linear combinations of the observed variables (i.e. the excitation-emission wavelengths, the inputs) that yield the best linear regression to the predicted variables (i.e. the biological parameters, the outputs). These linear combinations are known as Latent Variables (LVs), and can be seen as underlying structures or patterns that are correlated to the predicted parameters more directly than the original spectral variables measured by the experiment. More extensive descriptions of PLS can be found elsewhere ${ }^{25}$. In the current work, the multiway version of PLS, known as N-PLS ${ }^{26}$, was used, which allows for fully exploiting the mathematical relationships among the three modes of the EEM data (i.e. sample, excitation, emission).

To facilitate the modelling task, the predicted variables were converted into their logarithm with base 10 to normalise their distribution. The predictive models were validated by a 4 -fold double cross-validation ${ }^{27}$. In short, the data set was split randomly into a training and validation set, consisting of $75 \%$ and $25 \%$ of the total data, respectively. The training set was used to calibrate the model and optimise the number of LVs, using a leave-one-out cross-validation (LOOCV), in which one sample from the training set was removed, a PLS model was built on the remaining training samples and assessed for the left-out sample; this procedure was then repeated with a different leave-out sample until all samples have been rotated. The external validation set was held out of this loop and was used to validate the model with the optimal number of LVs as determined by the LOOCV. The whole procedure (LOOCV + external validation) was repeated three more times, using the external validation data that was previously used for training, until every sample has appeared in the external validation set once.

Several parameters were evaluated to assess model's quality: the variance explained in the biological parameters (\%), the root mean square error of cross-validation (RMSECV) and prediction (RMSEP), and the $\mathrm{R}^{2}$ and slopes of the training and external validation (from now on mentioned as validation) sets.

After evaluating the quality of the models, a final model was built for each predicted parameter using the whole data set, using the optimal number of LVs defined by the previous validation, in order to determine and examine the regression coefficient values.

All multivariate statistical analysis were performed in MATLAB (The MathWorks) using the drEEM toolbox (http://www.models.life.ku.dk/dreem) and n-way toolbox ${ }^{26,28}$.

Biological material. The strain Nannochloropsis oceanica NCT02 was kindly provided by Necton S.A. (Algarve, Potugal). Necton S.A. is willing to provide the strain on request.

\section{Results and Discussion}

Nannochloropsis oceanica production can aim at different final products. The whole biomass of microalgae is rich in pigments and fatty acids, but also has high valuable proteins and carbohydrates. Depending on the end-product desired, the production of the biomass can be tuned to reach higher yields. For that reason, the experiments of this work were designed to increase the concentration range of three different products, biomass (as cell concentration), chlorophyll and fatty acids. Having a wider range of scenarios increases the range of the outputs, which results in an increased strength of the prediction models. The information about the experimental conditions tested and the respective cell concentration, chlorophyll and fatty acids measurements are available in Supplementary Information.

Microalgae cultivation is characterised by low biomass concentrations, mainly to avoid dark zones in the bioreactors that can lead to lower photosynthetic efficiencies ${ }^{29}$. For this reason, microalgae samples have high water content, that results in the presence of high intensity Rayleigh scatter (Fig. 1a). The presence of water scatter would impact the estimation of the regression coefficients of the final models (presented in section 3.4$)^{30}$, and 

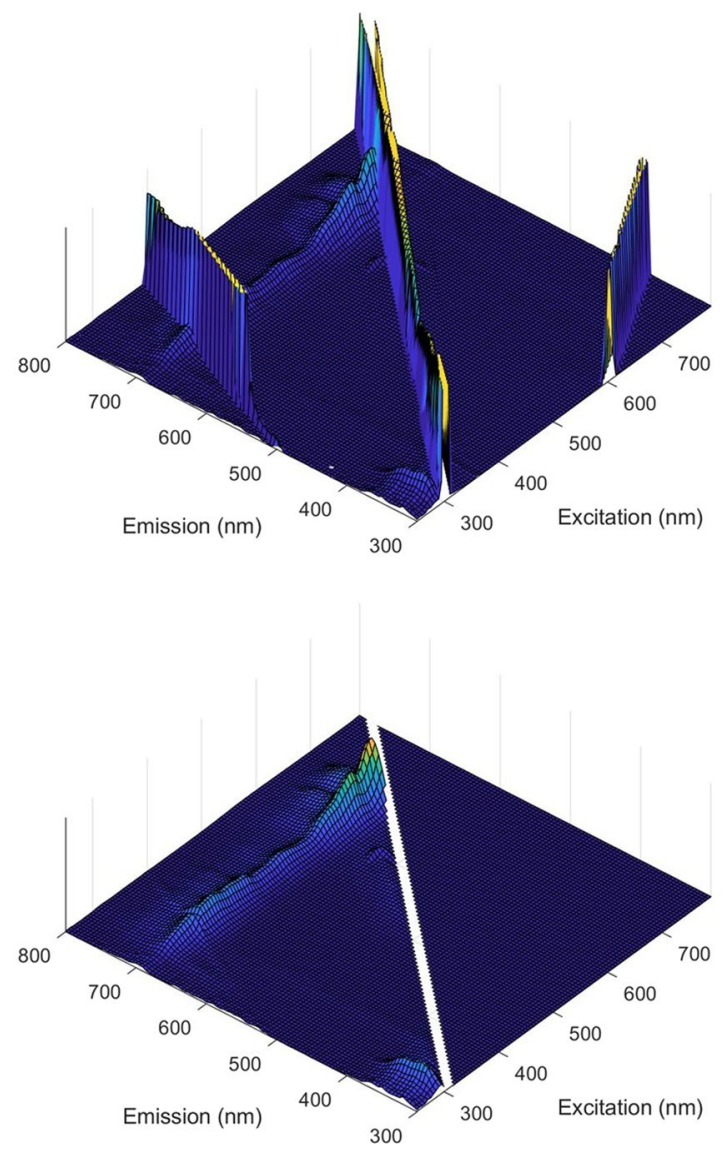

Figure 1. Fluorescence spectra of a Nannochloropsis oceanica sample: original spectra (a) and final spectra used as inputs in the PLS models (b). Rayleigh scatter of first order was removed and replaced by empty values; the second order was replaced with an interpolation of surrounding data points. Fluorescence signal corresponding to emission wavelengths ( $\mathrm{y}$-axis) shorter than the excitation wavelengths ( $\mathrm{x}$-axis) was replaced by zeros. Inner filter effects were also corrected whenever present.

since its signal is not proportional to the water content in the sample, it was removed before the PLS modelling, as described in Section 2.3. An example of the pre-processed spectra is shown in Fig. 1b.

Cell concentration. The model obtained to monitor cell concentration of $N$. oceanica can explain $84 \%$ of variance captured by the fluorescence spectroscopy (Fig. 2) with five LVs. A low root mean square error of $N$. oceanica concentration prediction (RMSEP) was observed $\left(0.27 \log _{10}\right.$ cells $\left./ \mathrm{mL}\right)$, which represents the average distance between the observed values and the ones predicted by the model. The relative error (in percentage), calculated as a quotient between the prediction error (RMSEP) and the observed cell concentration average value, is $3.18 \%$. The root mean square error of cross-validation (RMSECV) of $0.30 \log _{10}$ cells $/ \mathrm{mL}$ indicates absence of model overfit. The reported $\mathrm{R}^{2}$ for training refers to the model built on the whole training set, whereas the validation data was never used for any step of model building and therefore tends to have a lower $\mathrm{R}^{2}$. Furthermore, the observed lack-of-fit is mainly due to the poor predictions for samples with lowest cellular concentrations, see Fig. 2.

In the current industrial scenario, most microalgal products are sold as whole biomass powder, making total biomass a key parameter to control process efficiency. During cultivation at industrial scale, too high or too low biomass concentration can have an influence in several biological parameters. Low concentrations can result in an inefficient light absorption or photo inhibition, while high concentrations result in dark regions in the bioreactor triggering endogenous respiration ${ }^{29}$.

Biomass concentration can be monitored under different parameters, such as optical density (OD), dry weight $(\mathrm{g} / \mathrm{L})$ and cell concentration (cells/L). In the present work, biomass concentration was measured as cell concentration. The main reason was because the experiments were designed to give a wide range of cell concentration, but some experiments induce also other biological changes, such as different coloration or accumulation of fatty acids. For example, as mentioned by Janssen et al. ${ }^{31}$, accumulation of fatty acids in lipid bodies, due to nitrogen depletion medium, leads to an increase of the dry weight while the cell concentration reaches a plateau. This phenomenon was also observed with the experiments of this work (data not shown). Experiments using day/night cycle lead the biomass to follow circadian rhythms, which means that the cell size increases during the day while the cell concentration increases during the night (due to cell division) ${ }^{32,33}$. 


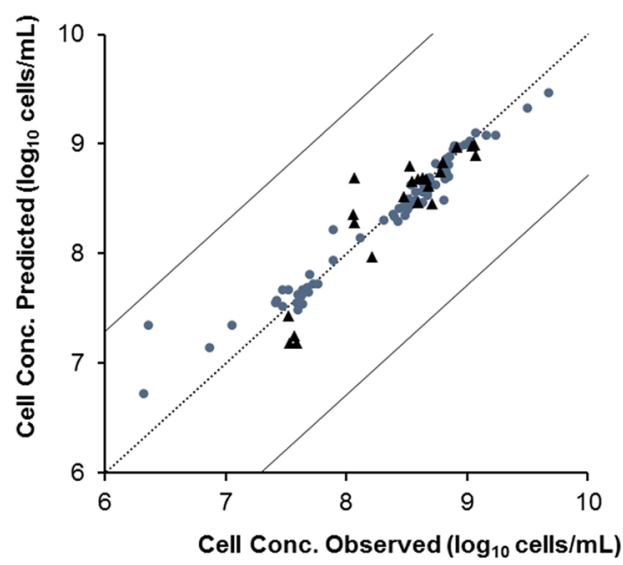

\begin{tabular}{|c|c|c|}
\hline Variance & $(\%)$ & 83.69 \\
\hline RMSECV & \multirow{2}{*}{$\left(\log _{10} c e / / \mathrm{s} / \mathrm{mL}\right)$} & 0.30 \\
\hline RMSEP & & 0.27 \\
\hline \multirow{2}{*}{ Training } & $\mathrm{R}^{2}$ & 0.88 \\
\hline & Slope & 0.85 \\
\hline \multirow{2}{*}{ Validation } & $\mathrm{R}^{2}$ & 0.66 \\
\hline & Slope & 0.88 \\
\hline \multicolumn{2}{|c|}{ Number of LVs } & 5 \\
\hline
\end{tabular}

Figure 2. Cell concentration prediction model (one of the four partitions of training/validation data sets). Training $(\boldsymbol{O})(\mathrm{n}=69)$ and validation $(\boldsymbol{\Delta})(\mathrm{n}=23)$ data are represented in $\log _{10}$ cells $/ \mathrm{mL}$. Model performance parameters: variance captured (Variance); root mean square error of cross-validation (RMSECV); root mean square error of prediction (RMSEP); coefficients of determination $\left(\mathrm{R}^{2}\right)$ and slopes of linear regression between observed and predicted data obtained respectively for the training and validation data sets; number of LVs used by the model.

A previous study reported the use of fluorescence spectroscopy to monitor cell concentration of a different microalgae, Dunaliella salina ${ }^{34}$. A slightly different modelling approach was used then, since PCA (principal component analysis) was performed on the EEMs, without the need to remove the scatter, prior to PLS modelling. Nevertheless, a similar explained variance was observed (between 85.7 and $86.3 \%$ ), with similar values of $\mathrm{R}^{2}$ for training and validation sets (between 0.82 and 0.86 ). These results, together with the results of this work, demonstrate the potential of using fluorescence spectroscopy as a monitoring tool for cell concentration with different microalgae biomass.

Chlorophyll. Chlorophyll is the most abundant light harvesting pigment in nature, enabling the photosynthesis, and is a molecule well-studied for its potential in several fields. In the feed and food supplements industry, chlorophyll is relevant for its anti-oxidant properties. Because of its bright green colour, it is also an appealing dye for the food and paint industries ${ }^{13,14}$.

Chlorophyll content in microalgae is tightly correlated with the light intensity and circadian rhythms. It was reported that chlorophyll content increases during the light period, and starts to decrease with the beginning of a dark period ${ }^{32,33,35}$. That phenomenon is explained by the fact that the cell division mechanism is favourable in the dark period, and since Nannochloropsis genus divide by binary fission, the chlorophyll content of the "adult" cell is divided by its new cells ${ }^{33}$.

The experimental conditions induced a considerable variability in the chlorophyll content. As mentioned previously, day/night cycles induce the chlorophyll to oscillate during the experiments. Moreover, microalgae are known for their ability to adapt their photosynthetic apparatus to different light conditions, a process called photoacclimation. High light intensities reduce chlorophyll content to protect the cell, while low intensities induced the photosynthetic apparatus to synthesise chlorophyll, to provide the cell with more light harvesting capacity ${ }^{31,36}$. Nitrogen starvation, however decreases chlorophyll content and increases carotenoid concentrations to equip the cell with stress defence mechanisms ${ }^{31,33}$.

The chlorophyll content studied in these experiments enabled the development of an accurate model (Fig. 3), with a relative error of $1.31 \%$ using five LVs. The validation and training sets show high $\mathrm{R}^{2}$ and low errors, both RMSECV and RMSEP.

The possibility of monitoring chlorophyll content online provides information about the physiological state of the microalgae at real time. With this knowledge it is possible to take decisions during the cultivation process, without the need to perform the time-consuming lab analysis that are usually required.

Lipids. Several microalgae are being studied for their potential to produce high content and/or high-quality lipids. Having in mind the different opportunities for an enriched-lipid biomass, the N. oceanica lipid profile was assessed through a set of different experiments. Nitrogen depletion is a well-documented strategy to increase lipid content in the TAG fraction ${ }^{10,37}$, while low light conditions were documented to lead to an increase in the cellular membranes ${ }^{37}$. Also, low temperatures were described to increase the content of unsaturated fatty acids ${ }^{10,38,39}$, whereas high temperature favour the saturated ${ }^{40}$.

Models were obtained to monitor fatty acids as total content (Fig. 4a), as well as saturated (Fig. 4b) and unsaturated (Fig. 4c) content only (Table 2). Values of explained variance ranged between 87 and $92 \%$. When compared with the previous models of this work, a higher number of LVs is needed (between 9 and 10). The relative error of prediction for total and unsaturated fatty acids was $5.89 \%$ and $5.62 \%$, respectively, lower than the value found for saturated fatty acids (9.54\%). All $\mathrm{R}^{2}$ of validation and training set were above 0.74 and slopes close to 1 .

When cultivated under optimal growing conditions, most of the microalgae lipids are present on the cellular membrane ${ }^{11}$. However, under stress growing conditions some microalgae, like Nannochloropsis, can accumulate up to $45 \%$ of their dry weight in triacylglycerol (TAG) ${ }^{10,37}$. According to the fraction of the cell where the lipid is 
Chlorophyll Observed $\left(\log _{10} \mathrm{mg} / \mathrm{cell}\right)$

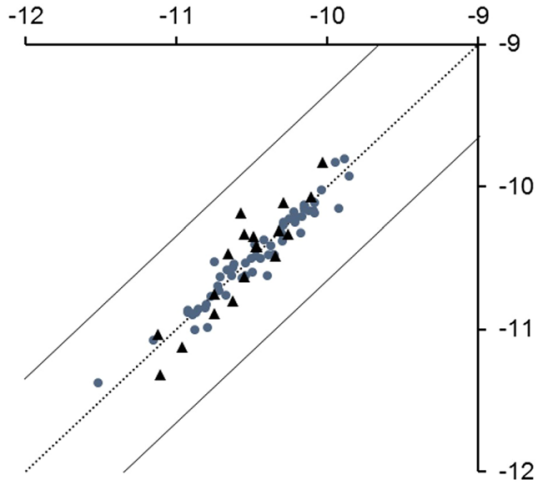

\begin{tabular}{|c|c|c|}
\hline Variance & $(\%)$ & 86.47 \\
\hline RMSECV & \multirow{2}{*}{$\left(\log _{10} \mathrm{mg} / \mathrm{cell}\right)$} & 0.39 \\
\hline RMSEP & & 0.32 \\
\hline \multirow{2}{*}{ Training } & $R^{2}$ & 0.88 \\
\hline & Slope & 0.97 \\
\hline \multirow{2}{*}{ Validation } & $\mathrm{R}^{2}$ & 0.78 \\
\hline & Slope & 0.97 \\
\hline \multicolumn{2}{|c|}{ Number of LVs } & 5 \\
\hline
\end{tabular}

Figure 3. Chlorophyll content prediction model (one of the four partitions of training/validation data sets). Training $(\mathbf{O})(\mathrm{n}=57)$ and validation $(\boldsymbol{\Lambda})(\mathrm{n}=19)$ data are represented in $\log _{10} \mathrm{mg} /$ cell. Model performance parameters: variance captured (Variance); root mean square error of cross-validation (RMSECV); root mean square error of prediction (RMSEP); coefficients of determination $\left(\mathrm{R}^{2}\right)$ and slopes of linear regression between observed and predicted data obtained respectively for the training and validation data sets; number of LVs used by the model.

\begin{tabular}{|l|l|l|l|l|}
\hline & & Total & Saturated & Unsaturated \\
\hline Variance & \multirow{2}{*}{$(\%)$} & 92.30 & 91.24 & 86.77 \\
\hline RMSECV & \multirow{2}{*}{$\left(\log _{10 \%} g / g_{D W}\right)$} & 0.21 & 0.29 & 0.17 \\
\cline { 3 - 5 } RMSEP & & 0.19 & 0.23 & 0.15 \\
\hline \multirow{2}{*}{ Training } & $\mathrm{R}^{2}$ & 0.87 & 0.90 & 0.85 \\
\cline { 2 - 5 } & Slope & 0.92 & 0.93 & 0.95 \\
\hline \multirow{2}{*}{ Validation } & $\mathrm{R}^{2}$ & 0.78 & 0.81 & 0.74 \\
\cline { 2 - 5 } & Slope & 0.84 & 0.95 & 0.99 \\
\hline \multirow{2}{*}{ Number of LVs } & 10 & 10 & 9 \\
\hline
\end{tabular}

Table 2. Prediction model parameters for total, saturated and unsaturated fatty acids. Model performance parameters: variance captured (Variance); root mean square error of cross-validation (RMSECV); root mean square error of prediction (RMSEP); coefficients of determination (R2) and slopes of linear regression between observed and predicted data obtained respectively for the training $(\mathrm{n}=54)$ and validation $(\mathrm{n}=18)$ data sets; number of LVs (latent variables) used by the model.

accumulated and its profile, the final destination of the lipid-enriched biomass can vary from feed or food supplements to biodiesel production ${ }^{11,41,42}$.

Fatty acids can be classified into saturated or unsaturated, the latter into mono- or polyunsaturated according to the number of double chemical bonds. Biomass produced with the aim of supplying feed or food supplements industries is desired to be rich in unsaturated fatty acids, preferably omega-3 fatty acids such as EPA (eicosapentaenoic acid) or DHA (docosahexaenoic acid) $)^{11,37}$. Biomass produced for biofuel applications needs to fulfil quality parameters such as ignition and combustion performance, and those are directly correlated with saturated and unsaturated content ${ }^{10,43}$.

To our knowledge, fluorescence spectroscopy was not previously reported as an online monitoring tool for lipid content. It is known that fluorescence spectroscopy is highly sensitive to detect the presence of natural fluorophores and that lipid molecules are not natural fluorophores. In the presence of a complex matrix, like microalgae cultivation broth, fluorescence spectroscopy is able not only to identify the natural fluorophores (intra or extracellular) but also the relations between these compounds and the non-fluorophores ${ }^{9}$. For that reason, fluorescence spectroscopy spectra cannot be directly used for quantification, but is a powerful tool revealing the correlations between compounds that emit natural fluorescence, the ones that capture it, and the ones that somehow mask the fluorescence signal.

Regression coefficients of the final models for cell concentration, chlorophyll and fatty acids. The experiments performed enabled to acquire a wide range of scenarios of a N. oceanica cultivation for different end products. After calibrating the models for the parameters cell concentration, chlorophyll and fatty acids, it is possible to confirm that fluorescence spectroscopy has a great potential for online monitoring of all three parameters simultaneously.

Aiming at the application of fluorescence spectroscopy, a final model was created for each parameter studied, using $100 \%$ of the data as training set. Figure 5 shows the regression coefficients obtained for each output, where 

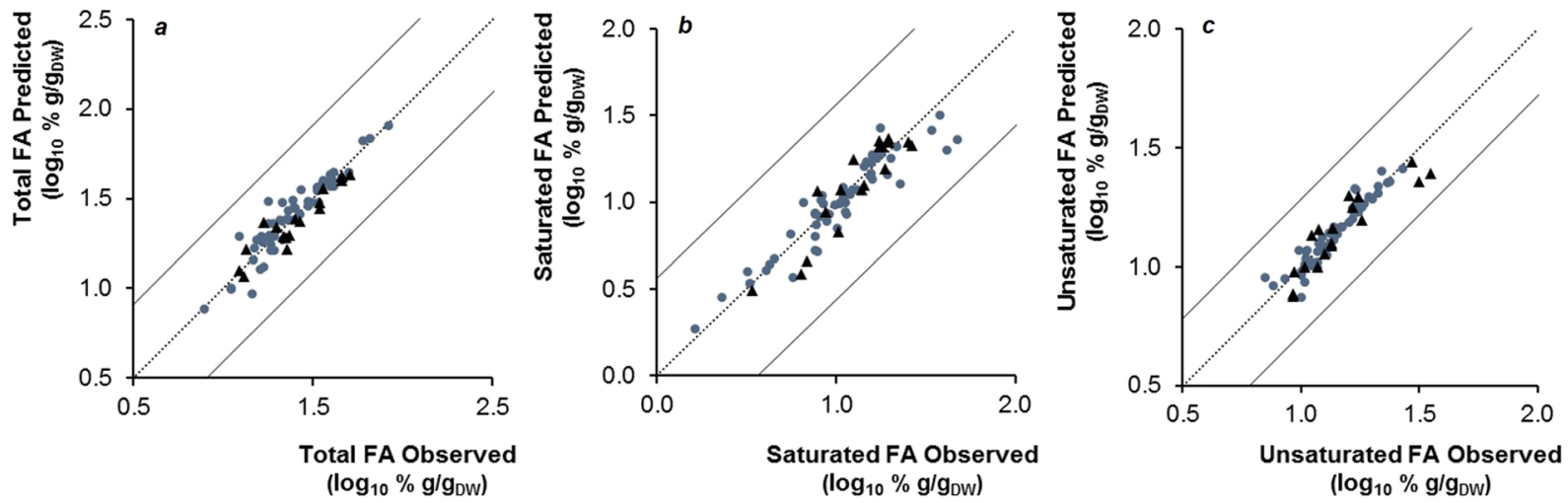

Figure 4. Fatty acids (FA) prediction models for total (a), saturated (b) and unsaturated (c) FA (one of the four partitions of training/validation data sets). Training $(\mathbf{O})(\mathrm{n}=54)$ and validation $(\boldsymbol{\Lambda})(\mathrm{n}=18)$ data are represented in $\log _{10} \% \mathrm{~g} / \mathrm{g}$ DW.
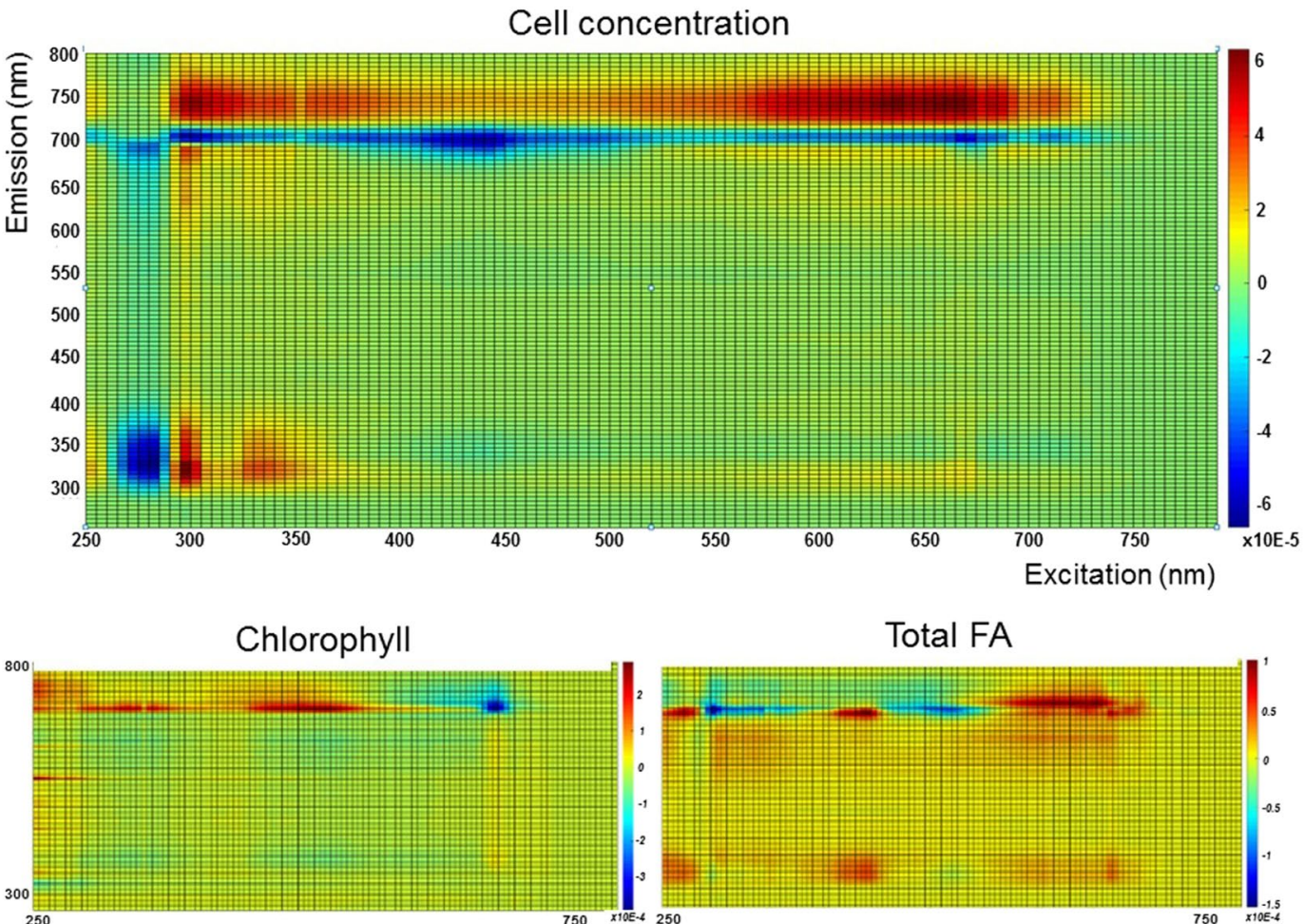

Total FA

Saturated FA

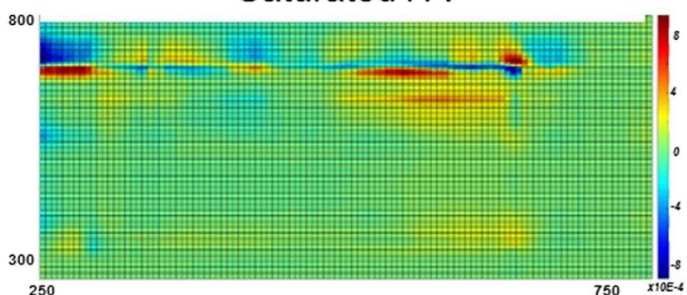

Unsaturated FA

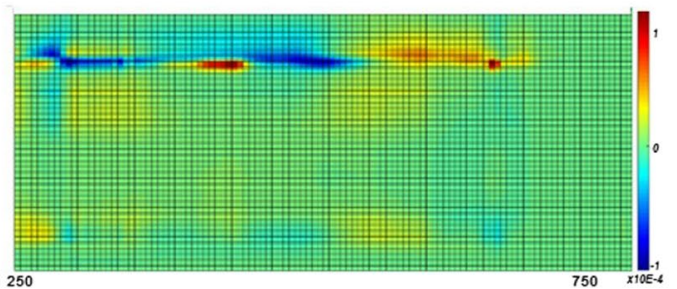

Figure 5. Regression coefficients of the prediction models for cell concentration, chlorophyll, and fatty acids (FA) as total, saturated and unsaturated. The training set used $100 \%$ of the data set. Excitation wavelengths are represented in the $\mathrm{x}$-axis, emission wavelengths in the $\mathrm{y}$-axis, and intensity is represented in the colour bar on the right side. 
the excitation and emission wavelengths (in $\mathrm{nm}$ ) are in the $\mathrm{x}$-axis and $\mathrm{y}$-axis, respectively, and each square is an excitation/emission pair. The weight of each regression coefficient is represented in colour-scale.

For the cell concentration model, two main regions can be distinguished as having relevant regression coefficient weight (positive or negative): a band at emission wavelengths higher than $600 \mathrm{~nm}$ (whole excitation wavelength range), and a region for excitation and emission wavelengths lower than $400 \mathrm{~nm}$. This reveals a similarity with the overall fluorescence signal of a sample, where these two regions have high fluorescence intensities. As described previously, these two regions of the spectra correspond to the pigments fluorescence band and the protein-like region (aromatic aminoacids), respectively ${ }^{34,44-46}$. Several differences can be noticed between the regression coefficients map of the cell concentration model and the remaining outputs. The protein-like region does not have the same weight as for cell concentration, and different weights are attributed to the pigments band. As expected, a high correlation is shown between the pigments band and the regression coefficient map for chlorophyll content prediction. Also, specific areas of this pigment band are used for fatty acids content prediction (as total, saturated or unsaturated). These results confirm the relationship previously described between chlorophyll and fatty acids content ${ }^{33}$, and that although fatty acids do not emit fluorescence, they interfere with the signal of natural fluorophores like chlorophyll. The development of a simpler spectrofluorometric technique, able to acquire signal in those two regions of the spectra, instead of the entire range, would possibly simplify the analysis by decreasing the acquisition time of each data point.

Using fluorescence spectroscopy to simultaneously monitor several biological parameters has been described as one of the powerful characteristics of this technique ${ }^{9}$. By coupling the fluorescence EEMs and the regression coefficients developed by chemometric models, more knowledge about the cultivation process can be acquired, enabling important decisions at real time, like the optimum harvesting time.

\section{Conclusions}

The present work demonstrates the feasibility of using fluorescence spectroscopy coupled to chemometrics to assess multiple parameters during N. oceanica cultivation. It was shown that this technique can be applied to this microalga to assess not only biomass (as cell concentration) and pigments (chlorophyll), as in previous studies, but also different fractions of fatty acids. This outcome has a major impact on the monitoring of microalgae production, especially when aiming lipids production.

Different environmental conditions were tested to increase the response range of the parameters under study, which allowed the development of accurate models for all the parameters with low errors (REMSECV and RMSEP between 0.12 and 0.40 ) and high $\mathrm{R}^{2}$ (between 0.65 and 0.93 ). Furthermore, the regression coefficient maps highlight the importance of the pigment and the protein regions for the development of these models.

In overall, the fluorescence excitation-emission matrices contain a huge amount of diverse information from the process that can be translated into quantitative information using adequate mathematical tools, such as developed in this study.

\section{Data availability}

The datasets generated and analysed during the current study will be available in the MAGNIFICENT Zenodo repository, after publication.

Received: 22 November 2019; Accepted: 31 March 2020;

Published online: 06 May 2020

\section{References}

1. Brasil, B. S. A. F., Silva, F. C. P. \& Siqueira, F. G. Microalgae biorefineries: The Brazilian scenario in perspective. N. Biotechnol. 39, 90-98 (2017)

2. Chua, E. T. \& Schenk, P. M. A biorefinery for Nannochloropsis: Induction, harvesting, and extraction of EPA-rich oil and high-value protein. Bioresour. Technol. 244, 1416-1424 (2017).

3. Ulber, R., Frerichs, J. G. \& Beutel, S. Optical sensor systems for bioprocess monitoring. Anal. Bioanal. Chem. 376, 342-348 (2003).

4. Lenhardt, L., Bro, R., Zeković, I., Dramićanin, T. \& Dramićanin, M. D. Fluorescence spectroscopy coupled with PARAFAC and PLS DA for characterization and classification of honey. Food Chem. 175, 284-91 (2015).

5. Ammari, F., Redjdal, L. \& Rutledge, D. N. Detection of orange juice frauds using front-face fluorescence spectroscopy and Independent Components Analysis. Food Chem. 168, 211-217 (2015).

6. Carstea, E. M., Bridgeman, J., Baker, A. \& Reynolds, D. M. Fluorescence spectroscopy for wastewater monitoring: A review. Water Res. 95, 205-219 (2016).

7. Galinha, C. F. et al. Real-time monitoring of membrane bioreactors with $2 \mathrm{D}$-fluorescence data and statistically based models. Water Sci. Technol. 63, 1381-8 (2011).

8. Galinha, C. F. et al. Multivariate statistically-based modelling of a membrane bioreactor for wastewater treatment using $2 \mathrm{D}$ fluorescence monitoring data. Water Res. 46, 3623-36 (2012).

9. Joseph R. Lakowicz. Principles of fluorescence spectroscopy. Principles of Fluorescence Spectroscopy, https://doi.org/10.1007/978-0387-46312-4 (2006)

10. Ma, X. N., Chen, T. P., Yang, B., Liu, J. \& Chen, F. Lipid production from Nannochloropsis. Mar. Drugs 14 (2016).

11. Li-Beisson, Y., Thelen, J. J., Fedosejevs, E. \& Harwood, J. L. The lipid biochemistry of eukaryotic algae. Prog. Lipid Res. 74, 31-68 (2019).

12. Chia, S. R. et al. Analysis of Economic and Environmental Aspects of Microalgae Biorefinery for Biofuels Production: A Review. Biotechnol. J. 13, 1-10 (2018).

13. Chew, K. W. et al. Bioresource Technology Microalgae biorefinery: High value products perspectives. Bioresour. Technol. 229, 53-62 (2017).

14. Rodrigues, D. B., Menezes, C. R., Mercadante, A. Z., Jacob-lopes, E. \& Zepka, L. Q. Bioactive pigments from microalgae Phormidium autumnale. FRIN 77, 273-279 (2015).

15. Sá, M. et al. Development of a monitoring tool based on fluorescence and climatic data for pigments profile estimation in Dunaliella salina. J. Appl. Phycol., https://doi.org/10.1007/s10811-019-01999-z (2019). 
16. Sá, M., Monte, J., Brazinha, C., Galinha, C. F. \& Crespo, J. G. Fluorescence coupled with chemometrics for simultaneous monitoring of cell concentration, cell viability and medium nitrate during production of carotenoid-rich Dunaliella salina. Algal Res. 44, 101720 (2019).

17. Sá, M., Monte, J., Brazinha, C., Galinha, C. F. \& Crespo, J. G. Fluorescence coupled with chemometrics for simultaneous monitoring of cell concentration, cell viability and medium nitrate during production of carotenoid-rich Dunaliella salina. Algal Res. 44, 101720 (2019).

18. Shin, Y.-H. et al. A portable fluorescent sensor for on-site detection of microalgae. Microelectron. Eng. 144, 6-11 (2015).

19. Sá, M. et al. Monitoring of eicosapentaenoic acid (EPA) production in the microalgae Nannochloropsis oceanica. Algal Res. 45 , $101766(2020)$.

20. Leu, K.-L. \& Hsu, B.-D. A programmed cell disintegration of Chlorella after heat stress. Plant Sci. 168, 145-152 (2005).

21. Breuer, G. et al. Superior triacylglycerol (TAG) accumulation in starchless mutants of Scenedesmus obliquus: (II) evaluation of TAG yield and productivity in controlled photobioreactors. Biotechnol. Biofuels 7, 70 (2014).

22. León-Saiki, G. M. et al. The role of starch as transient energy buffer in synchronized microalgal growth in Acutodesmus obliquus. Algal Res. 25, 160-167 (2017).

23. Bahram, M., Bro, R., Stedmon, C. \& Afkhami, A. Handling of Rayleigh and Raman scatter for PARAFAC modeling of fluorescence data using interpolation. J. Chemom. 99-105, https://doi.org/10.1002/cem.978 (2006).

24. Murphy, K. R., Stedmon, C. A., Graeber, D. \& Bro, R. Fluorescence spectroscopy and multi-way techniques. PARAFAC. Anal. Methods 5, 6557-6566 (2013).

25. Wold, S., Sjostrom, M. \& Eriksson, L. PLS-regression: a basic tool of chemometrics. Chemom. Intell. Lab. Syst. 58, 109-130 (2001).

26. Bro, R. Multiway calibration. Multilinear PLS. J. Chemom. 10, 47-61 (1996).

27. Filzmoser, P. Liebmann, B. \& Varmuza, K. Repeated double cross validation. J. Chemom. 23, 160-171 (2009).

28. Andersson, C. A. \& Bro, R. The N-way Toolbox for MATLAB. Chemom. Intell. Lab. Syst. 52, 1-4 (2000).

29. de Vree, J. H. et al. Turbidostat operation of outdoor pilot-scale photobioreactors. Algal Res. 18, 198-208 (2016).

30. Zepp, R. G., Sheldon, W. M. \& Moran, M. A. Dissolved organic fluorophores in southeastern US coastal waters: Correction method for eliminating Rayleigh and Raman scattering peaks in excitation-emission matrices. Mar. Chem. 89, 15-36 (2004).

31. Janssen, J. H. et al. Effect of nitrogen addition on lipid productivity of nitrogen starved Nannochloropsis gaditana. Algal Res. 33, $125-132(2018)$

32. de Winter, L., Klok, A. J., Cuaresma Franco, M., Barbosa, M. J. \& Wijffels, R. H. The synchronized cell cycle of Neochloris oleoabundans and its influence on biomass composition under constant light conditions. Algal Res. 2, 313-320 (2013).

33. Fábregas, J., Maseda, A., Domínguez, A., Ferreira, M. \& Otero, A. Changes in the cell composition of the marine microalga, Nannochloropsis gaditana, during a light:dark cycle. Biotechnol. Lett. 24, 1699-1703 (2002).

34. Sá, M., Monte, J., Brazinha, C., Galinha, C. F. \& Crespo, J. G. 2D Fluorescence spectroscopy for monitoring Dunaliella salina concentration and integrity during membrane harvesting. Algal Res. 24, 325-332 (2017).

35. Braun, R., Farré, E. M., Schurr, U. \& Matsubara, S. Effects of light and circadian clock on growth and chlorophyll accumulation of N annochloropsis gaditana. J. Phycol. 50, 515-525 (2014).

36. Dubinsky, Z. \& Stambler, N. Photoacclimation processes in phytoplankton: Mechanisms, consequences, and applications. Aquat. Microb. Ecol. 56, 163-176 (2009).

37. Janssen, J. H., Wijffels, R. H. \& Barbosa, M. J. Lipid Production in Nannochloropsis gaditana during Nitrogen Starvation. Biology (Basel). 8,5 (2019).

38. Jiang, H. \& Gao, K. Effects of lowering temperature during culture on the production of polyunsaturated fatty acids in the marine diatom Phaeodactylum tricornutum (Bacillariophyceae). J. Phycol. 40, 651-654 (2004).

39. Wang, Y., He, B., Sun, Z. \& Chen, Y. F. Chemically enhanced lipid production from microalgae under low sub-optimal temperature. Algal Res. 16, 20-27 (2016).

40. Ördög, V. et al. Effect of temperature and nitrogen concentration on lipid productivity and fatty acid composition in three Chlorella strains. Algal Res. 16, 141-149 (2016).

41. Draaisma, R. B. et al. Food commodities from microalgae. Curr. Opin. Biotechnol. 24, 169-177 (2013).

42. Caballero, M. J. et al. Morphological aspects of intestinal cells from gilthead seabream (Sparus aurata) fed diets containing different lipid sources. Aquaculture 225, 325-340 (2003).

43. Nascimento, I. A. et al. Microalgae Versus Land Crops as Feedstock for Biodiesel: Productivity, Quality, and Standard Compliance. Bioenergy Res. 7, 1002-1013 (2014).

44. Galinha, C. F. et al. Two-dimensional fluorescence as a fingerprinting tool for monitoring wastewater treatment systems. J. Chem. Technol. Biotechnol. 86, 985-992 (2011).

45. Ziegmann, M., Abert, M., Müller, M. \& Frimmel, F. H. Use of fluorescence fingerprints for the estimation of bloom formation and toxin production of Microcystis aeruginosa. Water Res. 44, 195-204 (2010).

46. Moberg, L., Robertsson, G. \& Karlberg, B. Spectrofluorimetric determination of chlorophylls and pheopigments using parallel factor analysis. Talanta 54, 161-170 (2001).

\section{Acknowledgements}

This work was supported by the project "Microalgae As a Green source from Nutritional Ingredients for Food/ Feed and Ingredients for Cosmetics by cost effective New Technologies" [MAGNIFICENT], funded by Biobased Industries Joint Technology Initiative under European Commission [project ID: 745754], by the Associate Laboratory for Green Chemistry - LAQV which is financed by national funds from FCT/MCTES (UID/ QUI/50006/2019), and by the PhD grant SFRH/BD/108894/2015 of FCT/MCTES.

\section{Author contributions}

M.S., C.B., N.F.L., J.J., R.W., J.G.C., M.B. and C.F.G. were involved in the design of experiments. M.S. and N.F.L. worked in the results acquisition. M.S. and C.B. were involved on the creation of a new code for the models development. The results obtained were analysed and discussed between M.S., C.B., N.F.L., J.J., R.W., J.G.C., M.B. and C.F.G.. M.S. prepared the draft of the manuscript and C.B., N.F.L., J.J., R.W., J.G.C., M.B. and C.F.G. contributed with a critical revision. The main funding for this study was obtained by M.B. Final approval of the article was done by all authors.

\section{Competing interests}

The authors declare no competing interests.

Additional information

Supplementary information is available for this paper at https://doi.org/10.1038/s41598-020-64628-7. 
Correspondence and requests for materials should be addressed to C.F.G.

Reprints and permissions information is available at www.nature.com/reprints.

Publisher's note Springer Nature remains neutral with regard to jurisdictional claims in published maps and institutional affiliations.

(c) Open Access This article is licensed under a Creative Commons Attribution 4.0 International License, which permits use, sharing, adaptation, distribution and reproduction in any medium or format, as long as you give appropriate credit to the original author(s) and the source, provide a link to the Creative Commons license, and indicate if changes were made. The images or other third party material in this article are included in the article's Creative Commons license, unless indicated otherwise in a credit line to the material. If material is not included in the article's Creative Commons license and your intended use is not permitted by statutory regulation or exceeds the permitted use, you will need to obtain permission directly from the copyright holder. To view a copy of this license, visit http://creativecommons.org/licenses/by/4.0/.

(C) The Author(s) 2020 